\title{
Metodología combinada para entender la duración del amamantamiento en barrios pobres de Managua, Nicaragua ${ }^{1}$
}

\author{
Janet Irene Picado, ${ }^{2}$ Christine M. Olson ${ }^{2}$ y \\ Kathleen M. Rasmussen ${ }^{2}$
}

RESUMEN Se examinó la relación de diversas variables maternas con la duración del amamantamiento exclusivo y con la duración total del amamantamiento y se estudiaron las actitudes, percepciones y creencias de las mujeres de los barrios pobres de Managua, Nicaragua, respecto a la lactancia natural. El trabajo de campo se realizó con métodos cualicuantitativos en diciembre de 1992 y enero de 1993. Se entrevistó con un cuestionario estructurado a 556 madres de niños menores de 12 meses y se reunieron cuatro grupos de discusión dirigida en los que participaron 20 madres. A la semana de edad casi todos los hijos de las madres que participaron en la encuesta habían sido amamantados, pero solo $45 \%$ habían recibido amamantamiento exclusivo. A las 12 semanas 30\% ya estaban totalmente destetados. Los grupos de discusión revelaron la coexistencia de valoraciones positivas tanto del amamantamiento como de la alimentación con biberón. El amamantamiento exclusivo se consideró perjudicial para la madre, y la leche materna, insuficiente para mantener al niño. La experiencia previa guardó una relación importante con la duración del amamantamiento exclusivo y con el período total de amamantamiento. Las actitudes, el apoyo social y la situación de trabajo fueron importantes factores predictivos de la duración total del amamantamiento. En general, los resultados de las entrevistas y de los grupos de discusión fueron coherentes y muestran que para promover la lactancia natural exclusiva se necesitan apoyo social, un ambiente comunitario favorable al amamantamiento y políticas para abordar los problemas de las madres trabajadoras. Los métodos de investigación complementarios fueron útiles para obtener información sobre la importancia relativa de diferentes factores relacionados con la duración del amamantamiento y entender más a fondo esa práctica desde el punto de vista materno.

Los especialistas en salud y nutrición $(1,2)$ recomiendan como patrón ideal de alimentación del lactante un período de 4 a 6 meses de amaman-

\footnotetext{
Una versión previa de este trabajo fue presentada por la primera autora para su grado de Master of Science (Maestría en Ciencias).

2 Universidad Cornell, División de Ciencias de la Nutrición, Ithaca, Nueva York. La correspondencia debe enviarse a la segunda autora a la siguiente dirección postal: Division of Nutritional Sciences, Martha Van Rensselaer Hall, Cornell University, Ithaca, New York, 14853 EUA.
}

tamiento exclusivo (es decir, leche materna como único alimento o bebida) y amamantamiento no exclusivo al menos hasta los 12 meses. En Managua, Nicaragua, casi todas las mujeres amamantan a sus hijos, pero solo una pequeña proporción de madres practica el amamantamiento exclusivo y muchas destetan al hijo antes de los 3 meses de edad (3-6). Este patrón de alimentación tiene consecuencias desfavorables para la salud infantil y es un grave problema en un país donde las enfermedades diarreicas son la causa principal de morbilidad y mortalidad en la infancia (7).

El amamantamiento materno o lactancia natural debe ser posible para todas las madres y todos los nacidos. Es preciso proteger, apoyar y promover esa práctica, particularmente entre las mujeres pobres del sector urbano. Este estudio fue motivado por la necesidad de obtener información para planear intervenciones comunitarias de apoyo a la lactancia natural. 
En su artículo de revisión, Allen y Pelto (8) clasificaron las variables que se consideran determinantes de la duración del amamantamiento. Factores socioconductuales tales como las actitudes, el apoyo social y la situación laboral, se denominan variables "de origen ambiental (cultural) o sicológico". Las variables bioculturales son aquellas que tienen "un efecto mediado probablemente por interacciones de los procesos biológicos con los socioconductuales", como la experiencia previa, las prácticas de alimentación en el hospital, la idea que tiene la madre de la suficiencia de la leche, el horario de alimentación, etc. Partiendo del marco conceptual de Allen y Pelto (8), para el presente estudio se hizo una revisión de las publicaciones sobre amamantamiento en Nicaragua $\mathrm{u}$ otros países en las que se hubieran incluido variables socioconductuales y bioculturales. Se desarrolló así un marco conceptual con las variables más verosímilmente relacionadas con la duración del amamantamiento en Nicaragua. Dichas variables, que orientaron la investigación, fueron las siguientes características maternas: actitudes con respecto al amamantamiento, apoyo social, situación laboral, experiencia previa de amamantamiento, uso de los servicios de salud, autoeficacia (véase más adelante su definición) y subsidios del sistema de seguridad social para adquirir leche.

El estudio se llevó a cabo con una metodología combinada a base de una encuesta y grupos de discusión dirigida. El principio básico de este diseño es la "complementariedad", es decir, el uso de métodos cualitativos y cuantitativos para estudiar de una forma más completa el fenómeno de interés (9). La encuesta permitió hacer estimaciones cuantitativas de la duración del amamantamiento y aplicar técnicas estadísticas multifactoriales para estudiar la relación de ciertas variables con la duración del amamantamiento exclusivo y con la duración total del amamantamiento, es decir, el período comprendido desde el nacimiento hasta el destete completo del lactante. Los grupos de discusión dirigida o "grupos focalizados" (focus groups en inglés) brindaron elementos para una mejor interpretación de los resultados de la encuesta cuantitativa.

La investigación intentó responder a las siguientes preguntas: 1) Cuando el amamantamiento exclusivo es breve ¿lo es también el período total de amamantamiento? 2) ¿Tienen relación con una mayor duración del amamantamiento las actitudes favorables, la sensación de autoeficacia, el subsidio para la compra de leche, el trabajo en el hogar, el apoyo social, la experiencia previa y el contacto con los servicios de salud? 3) ¿Qué prácticas de alimentación maternoinfantil y qué actitudes, ideas y creencias constituyen barreras para el amamantamiento exclusivo y llevan al destete prematuro? La encuesta sirvió para plantear las dos primeras preguntas; la tercera se abordó en los grupos de discusión. El protocolo del estudio fue aprobado por el Comité sobre Investigaciones con Seres Humanos de la Universidad Cornell.

\section{MATERIALES Y MÉTODOS}

La población de estudio se compuso de madres con hijos menores de un año que residían en los barrios pobres de Managua. El marco muestral fue una lista de 159 barrios de los seis distritos de Managua clasificados como "populares" o como asentamientos espontáneos por el municipio de Managua. Se estima que alrededor de $25 \%$ de la población de la ciudad vive en esos barrios.

Para seleccionar la muestra se escogieron mediante dígitos aleatorios producidos por computador 15 barrios de los seis distritos geográficos de Managua. Sin embargo, una vez estimada la población de lactantes de esos barrios, fue obvio que eran demasiados para el estudio. Por tanto, se volvieron a seleccionar aleatoriamente algunos barrios del grupo inicial hasta obtener una muestra de aproximadamente 500 lactantes. En la muestra final se incluyeron nueve barrios (Carlos Reyna, Francisco Meza, Carlos Fonseca, Edmundo Matamoros, CubaRubén Darío, Pista Larreynaga, Las
Lomas, Villa Democracia y Eduardo Contreras), en cada uno de los cuales se hizo un muestreo completo de las madres con hijos menores de un año. De un total de 599 mujeres que fueron visitadas en su casa, se hicieron entrevistas a $556(93 \%)$. Se negaron a participar 12 mujeres y 31 no pudieron ser localizadas. Se excluyeron de la muestra las madres con lactantes adoptados o las que estaban fuera de la casa en el momento de la visita y no habían regresado al cabo de 3 días. Cuando se trataba de gemelos, se registraron en la entrevista solo los datos correspondientes a uno de ellos. De las 556 mujeres que fueron entrevistadas, no todas proporcionaron información sobre todas las variables.

Durante el mes de diciembre de 1992 las madres fueron entrevistadas en sus domicilios por personas capacitadas que utilizaron un cuestionario estructurado previamente codificado. El cuestionario, de 77 preguntas, se sometió a estudio piloto 2 meses antes con una muestra de 30 mujeres. Los dos resultados medidos fueron la duración del amamantamiento exclusivo y el período total de amamantamiento. La duración de este último período fue o bien la edad del niño (en semanas) en el momento de la entrevista cuando las mujeres todavía lo estaban amamantando, o bien el número de semanas desde el parto hasta el cese completo del amamantamiento cuando el niño ya estaba destetado. La duración del amamantamiento exclusivo se registró de forma similar. Las observaciones de las madres que todavía estaban amamantando se consideraron "censuradas" a efectos del análisis.

La duración del amamantamiento exclusivo se usó como variable explicativa continua. La experiencia materna en amamantamiento fue una variable categórica con cuatro categorías: primíparas, multíparas que no habían amamantado antes, multíparas que habían amamantado un máximo de 5 meses y multíparas con experiencia de un mínimo de 6 meses de amamantamiento.

La frecuencia de contacto con los servicios de salud se determinó preguntando a las madres si habían recibido atención prenatal y si el niño 
había nacido en el hospital. Las mujeres que no habían tenido ningún contacto o que habían tenido solo uno de los dos tipos de contacto se clasificaron en el grupo con menor contacto. Las que habían tenido ambos tipos de contacto se clasificaron en el grupo con más contacto.

Se preguntó a las madres si habían recibido un subsidio para la compra de leche del sistema de seguridad social (sí o no) y si trabajaban fuera de su casa (sí o no).

El apoyo social se determinó con dos variables categóricas diferentes: el tipo de familia de la madre (nuclear o extensa) y la percepción de la madre de haber sido (o no) apoyada en dar el pecho a su hijo por las personas más allegadas a ella. Se consideró que habían tenido mayor apoyo social las madres que creían haberlo recibido y las pertenecientes a una familia extensa.

Dos variables explicativas, autoeficacia y actitudes, se obtuvieron a partir de escalas basadas en cuestionarios. La teoría del aprendizaje social de Bandura define la autoeficacia (selfefficacy en inglés) como "la creencia en la capacidad propia para llevar a cabo una acción deliberada específica en una situación" $(10,11)$. La escala de autoeficacia utilizada fue desarrollada por Engle para evaluar la opinión de cada mujer respecto a su capacidad para actuar como madre y dar la atención correspondiente a su hijo. Esta escala ya se había usado antes en Managua (12). En nuestro estudio se pidió a la madre que se autocalificara en cada una de las 13 preguntas de la escala en comparación con otras dos mujeres adultas similares a ella, como una amiga, hermana o vecina. La puntuación total fue el número de veces que la madre se calificó como la mejor y una puntuación mayor significó mayor autoeficacia. El estadístico $\alpha$ de Cronbach de la escala fue 0,72 (13).

Las actitudes hacen referencia al sentimiento general de agrado o desagrado de una persona respecto a algo (14). La escala de actitudes que usamos fue la de un estudio hecho en la ciudad de México, convenientemente modificada (15). Por medio de ensayos piloto y de consultas con profesionales expertos se aseguró que las preguntas fueran culturalmente adecuadas y redactadas en un lenguaje comprensible. Se hicieron preguntas referentes a la facilidad del amamantamiento como método de alimentación, a la comodidad de la madre en cuanto a dar el pecho en público, al efecto del amamantamiento en el compañero o marido $\mathrm{y}$ en otros familiares $\mathrm{y}$ al efecto del método de alimentación en la salud y bienestar de la madre y del niño. Cada pregunta admitía dos respuestas: "estoy de acuerdo" y "no estoy de acuerdo". La puntuación total fue la suma de los puntos asignados a cada pregunta, una vez cambiados los códigos de las preguntas redactadas en términos negativos. Las preguntas mantenidas en la escala final utilizada en el análisis fueron las que dieron los valores máximos de $\alpha$. Esta escala tuvo un valor $\alpha$ de Cronbach de 0,55, que se consideró aceptable.

Se registraron también diversas características sociodemográficas de la madre que podrían estar relacionadas con los resultados y con las principales variables explicativas. Se registró la edad de la madre (en años), el lugar de nacimiento (Managua u otro), el nivel de escolaridad (analfabeta, instrucción elemental, secundaria o superior), el estado civil (con o sin compañero) y el riesgo ambiental. Esta variable se adaptó de estudios hechos en la ciudad de México (15) y en Managua (12). Se refiere al medio físico del hogar y se usó como medida de las condiciones socioeconómicas. Características del ambiente doméstico tales como los materiales con que está construida la vivienda, el techo, el piso, la localización de la fuente de agua, la evacuación de las aguas residuales, la fuente de electricidad, etc., se clasificaron según categorías previamente determinadas. Se adjudicaron puntuaciones a estas características y se obtuvo así un índice tanto mayor cuanto más precarias eran las condiciones de la vivienda y más pobre era la madre.

La duración del amamantamiento exclusivo y del período total de amamantamiento se analizó con el método de Kaplan-Meier de análisis de super- vivencia $(16,17)$. Los modelos predictivos de la duración del amamantamiento exclusivo y del período general de amamantamiento se elaboraron a partir del modelo de riesgo instantáneo proporcional de Cox (18). En este modelo se supone que los riesgos para distintos valores de la variable explicativa sean proporcionales. Este supuesto se sometió a prueba para cada variable explicativa mediante un análisis estratificado de "supervivencia" —es decir, el mantenimiento del amamantamiento- en el que se empleó la variable en cuestión para definir los estratos, sin incluirla en el análisis. Las curvas de supervivencia resultantes se examinaron para determinar si había proporcionalidad y se consideraron aceptables.

Para determinar qué variables tenían relación con los resultados se realizaron primero análisis unifactoriales. Se efectuaron también análisis multifactoriales para cada resultado, incluyendo todas las variables en el modelo y eliminándolas posteriormente una a una hasta llegar al modelo final. La selección se basó en la importancia de cada variable en el marco conceptual, su contribución a la comprensión del resultado estudiado, el valor $P$ correspondiente al coeficiente de regresión y los resultados del análisis unifactorial. Se utilizó el estadístico de Wald para determinar la significación estadística de cada coeficiente de regresión (18). Según los supuestos del estudio, se realizaron pruebas unilaterales con las variables explicativas principales y pruebas bilaterales con las demás.

El análisis inicial exploratorio de las relaciones entre las variables explicativas se llevó a cabo mediante los valores de ji cuadrado $\left(\chi^{2}\right)$ y del coeficiente de correlación de Pearson. Se analizaron los datos con los programas Epi Info Versión 5.01 B (19), SPSS/PC versión + 4.0 (20), sYSTAT for Windows, versión 5 (21) y el módulo SYSTAT SURVIVAL (22).

Se seleccionaron las participantes en los grupos de discusión con la ayuda de dirigentes comunitarios de cuatro barrios populares seleccionados por su cercanía al lugar donde habrían de celebrarse las reuniones. En cada barrio el dirigente comunitario preparó una 
lista de mujeres elegibles y se seleccionaron al azar de una lista 10 madres que fueron invitadas a asistir. Los criterios de inclusión fueron iguales que en la encuesta. De las 40 mujeres seleccionadas e invitadas a participar en un grupo, 20 participaron en las cuatro reuniones.

Los grupos de discusión se reunieron en enero de 1993 en el Centro de Investigación y Estudios de la Salud. Cada sesión duró aproximadamente una hora y se registró en un sistema de sonido. Para orientar la reunión se usó un guión de preguntas que había sido sometido a un ensayo piloto.

Se prepararon transcripciones textuales de las discusiones de los grupos. Cada entrevista se atomizó exhaustivamente hasta llegar a los segmentos mínimos de información útil. Más tarde estos segmentos se organizaron en categorías.

Para garantizar la calidad de los datos se emplearon varias técnicas basadas en el criterio de credibilidad de Guba y Lincoln (23). Este criterio se refiere al grado en que los datos reflejan la experiencia o el punto de vista émico ${ }^{3}$ del grupo en cuestión. Al concluir la entrevista de cada grupo focal, se presentó un breve resumen a las participantes. Posteriormente los moderadores titular y auxiliar comentaron la reunión. Las autoras de este artículo tienen varios años de experiencia de trabajo con mujeres de barrios similares a los del estudio, factor que debe favorecer la calidad de los datos.

\section{RESULTADOS}

La media de edad de las encuestadas $(n=555)$ fue de 24,7 años, con una desviación estándar (DE) de 5,8 y un recorrido de 15 a 48 años. El riesgo ambiental medio $(n=555)$ fue de 14,5 puntos (DE $=4,0$; recorrido, 7 a 23$)$. $\mathrm{La}$

\footnotetext{
3 En inglés el término emic se ha usado para referirse a conceptos o "constructos" que tienen significado solo en un contexto cultural concreto, en oposición a etic que se referiría a conceptos considerados universales y que han sido hallados en todas las culturas (R. M. Goldenson, Dictionary of psychology and psychiatry, Nueva York, Longman, 1984, pp. 254 y 279) (N. del editor).
}

puntuación media de actitud frente al amamantamiento $(n=556)$ fue 9,8 puntos (DE $=1,5$; recorrido, 7 a 14$)$. La media de puntuación de autoeficacia $(n=550)$ fue de $8,0(\mathrm{DE}=3,0$; recorrido, 0 a 13).

En el momento de la entrevista 338 de las mujeres encuestadas (61\%) amamantaban a sus hijos, pero solo $17 \mathrm{mu}$ jeres (3\%) les daban amamantamiento exclusivo. De las 218 (39\%) que no estaban amamantando a su hijo, 177 $(32 \%)$ le habían dado el pecho en semanas anteriores y $41(7 \%)$ no lo habían amamantado nunca.

Solo 61 madres de la muestra $(11 \%)$ eran analfabetas. La mayoría convivían con compañeros o maridos y eran amas de casa (cuadro 1).

La media de edad de las 20 madres participantes en los grupos de discusión fue de 25 años (DE $=6,0$; recorrido, 17 a 35) y similar a la de las encuestadas. Solo cinco eran primíparas y 10 de las 20 estaban amamantando a su hijo en el momento de la reunión.

Las madres que participaron en los grupos de discusión mencionaron muchos aspectos favorables del amamantamiento en general, aunque no señalaron ningún aspecto beneficioso concreto del amamantamiento exclusivo. También se observaron actitudes favorables tanto hacia el amamantamiento, como hacia la alimentación con biberón y el uso de otras clases de leche (cuadro 2).

El único incentivo mencionado por las madres para dar a sus hijos leche materna exclusivamente fue el ahorro, lo cual implica que las madres tienen una opinión desfavorable del amamantamiento exclusivo. Como explicó una de ellas: "A todos mis niños les he dado el pecho ... . [una] no tiene de dónde . . . para comprar el tarro de leche . . . ese es el motivo, pues, que le he dado . . . el pecho a mi niño ...".

En los grupos de discusión se pusieron de manifiesto actitudes y barreras sicológicas que dificultan el amamantamiento exclusivo: la creencia de que la leche materna no es suficiente para mantener al bebé y la idea de que un amamantamiento excesivo puede debilitar a la madre. Las ideas de la madre respecto a su propia alimentación

CUADRO 1. Características de una muestra de 556 madres de niños menores de un año, residentes en barrios pobres de Managua, Nicaragua, 1993

\begin{tabular}{|c|c|c|c|}
\hline Característica & Categoría & No. & $\%$ \\
\hline Escolaridad & $\begin{array}{l}\text { Analfabeta } \\
\text { Sabe leer y escribir o tiene instrucción elemental } \\
\text { Secundaria o superior }\end{array}$ & $\begin{array}{r}61 \\
230 \\
265\end{array}$ & $\begin{array}{l}11 \\
41 \\
48\end{array}$ \\
\hline Estado civil & $\begin{array}{l}\text { Soltera } \\
\text { Con compañero o casada }\end{array}$ & $\begin{array}{r}90 \\
466\end{array}$ & $\begin{array}{l}16 \\
84\end{array}$ \\
\hline Lugar de nacimiento & $\begin{array}{l}\text { Managua } \\
\text { Otro }\end{array}$ & $\begin{array}{l}319 \\
237\end{array}$ & $\begin{array}{l}57 \\
43\end{array}$ \\
\hline $\begin{array}{l}\text { Experiencia previa } \\
\text { en amamantamiento }\end{array}$ & $\begin{array}{l}\text { Ninguna (primípara) } \\
\text { Ninguna (multípara) } \\
\text { Amamantamiento previo }<6 \text { meses } \\
\text { Amamantamiento previo } \geq 6 \text { meses }\end{array}$ & $\begin{array}{r}169 \\
38 \\
121 \\
226\end{array}$ & $\begin{array}{r}31 \\
7 \\
22 \\
41\end{array}$ \\
\hline $\begin{array}{l}\text { Apoyo social } \\
\text { Clase de familia }\end{array}$ & $\begin{array}{l}\text { Nuclear } \\
\text { Extensa }\end{array}$ & $\begin{array}{l}290 \\
266\end{array}$ & $\begin{array}{l}52 \\
48\end{array}$ \\
\hline $\begin{array}{l}\text { Apoyo percibido } \\
\text { (para amamantar) }\end{array}$ & $\begin{array}{l}\text { Sí } \\
\text { No }\end{array}$ & $\begin{array}{l}368 \\
157\end{array}$ & $\begin{array}{l}70 \\
30\end{array}$ \\
\hline Situación laboral & $\begin{array}{l}\text { Con trabajo } \\
\text { Ama de casa }\end{array}$ & $\begin{array}{l}129 \\
427\end{array}$ & $\begin{array}{l}23 \\
77\end{array}$ \\
\hline Subsidio para leche & $\begin{array}{l}\text { Sí } \\
\text { No }\end{array}$ & $\begin{array}{r}84 \\
472\end{array}$ & $\begin{array}{l}15 \\
85\end{array}$ \\
\hline
\end{tabular}


CUADRO 2. Percepciones, actitudes y creencias respecto al amamantamiento y la introducción de otros productos en la alimentación del lactante. Resumen de las reuniones de discusión dirigida con 20 madres de menores de un año de barrios pobres de Managua, Nicaragua, 1993

\begin{tabular}{cc}
\hline Conducta o práctica & Factores de motivación \\
\hline Amamantamiento en general & - Las madres conocen sus beneficios: protege \\
(ventajas e inconvenientes). & contra la enfermedad, es más económico y \\
& es un método para espaciar los nacimientos. \\
- Las madres son animadas a amamantar por & sus propias madres y por otras mujeres con \\
& experiencia; también por médicos y \\
enfermeras. & Algunas madres creen que los lactantes \\
rechazan el biberón porque prefieren el \\
pecho.
\end{tabular}

Amamantamiento exclusivo.
Alimentación previa al amamantamiento:

- Purga con aceite y miel.

- Alimentación con leche en biberón al poco de nacer.

Suplementación de la leche materna a partir del primer mes con agua, bebidas de frutas $y$ leche en biberón.

Destete precoz.
Los mismos que en el apartado anterior
- Hay que limpiar o "purgar" el sistema digestivo del bebé.

- La madre cree que el lactante tiene hambre y necesita leche mientras a ella "le viene" la suya.

- La leche materna sola no permite mantener a un bebé.

- Como la madre tendrá que salir a trabajar, hay que enseñar al lactante a aceptar el biberón.
Obstáculos para el cambio

Actitudes negativas

- El estrés puede perjudicar el amamantamiento.

- El enfado de la madre puede hacer que el lactante enferme.

- Las madres enfermas deben dejar de dar el pecho.

Obstáculos materiales

- La madre trabaja fuera del hogar.

- El amamantamiento es doloroso: las madres tienen grietas y dolor en los pezones, dolor de espalda y fiebre.

Actitudes negativas

- La leche materna sola no es suficiente para un bebé.

- Se cree que el amamantamiento exclusivo puede hacer que la madre adelgace $y$ enferme.

- Se cree que la alimentación de la madre es deficiente por su situación económica.

- Se cree que alimentar al bebé solo con leche materna es indicio de dificultad económica.

Obstáculos materiales

- Trabajo fuera del hogar.

- La necesidad de alimentar a menudo al bebé dificulta las tareas domésticas.

- Las madres valoran las preparaciones para lactantes y la leche entera en polvo.

- Algunas madres creen que el lactante prefiere el biberón al pecho.

- Como en el apartado anterior. también pueden ser un obstáculo. Si la madre considera deficiente su alimentación, puede creer que no debe amamantar a sus hijos porque su leche no sirve. En palabras de una de las madres, “. . . como yo daba de amamantar a mi hija y tengo una pésima alimentación, entonces yo creo que esa leche no le va a servir a ella, porque solo agua y agua no le sirve".

El análisis de supervivencia referente a la duración del amamantamiento (figura 1) solo incluyó a los lactantes amamantados alguna vez. La mayoría de los niños (97\%) amamantaban a la semana de nacer, pero ya a esa edad solo la mitad recibía leche materna exclusivamente. A las 12 semanas, la proporción de amamantados se había reducido a cerca de $70 \%$ y solo $4 \%$ recibían amamantamiento exclusivo.

En el análisis unifactorial las variables relacionadas con una mayor duración del amamantamiento exclusivo fueron una puntuación alta de riesgo ambiental, la falta de subsidio para la compra de leche y la experiencia previa de haber amamantado a otro niño durante un mínimo de 6 meses. En el modelo final resultante del análisis multifactorial solo la experiencia previa guardó una relación significativa con el amamantamiento exclusivo (cuadros 3 y 4 ).

En los modelos unifactoriales la duración total del amamantamiento 
FIGURA 1. Análisis de supervivencia de la duración del amamantamiento

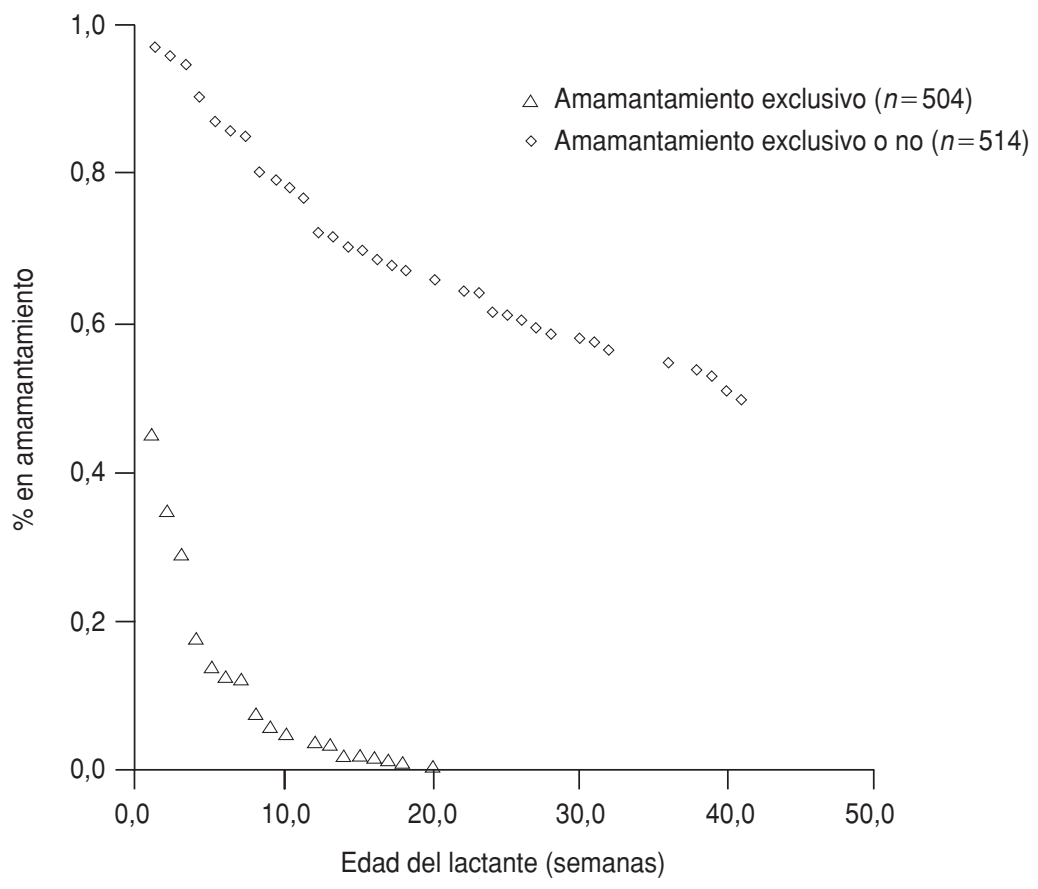

guardó relación con la duración del amamantamiento exclusivo, el grado de escolaridad (nivel secundario o superior), la experiencia previa de amamantamiento (6 meses o más), las actitudes, la situación laboral y el apoyo social (una familia extensa y la percepción de haber recibido apoyo) (cuadro 5).

El modelo final multifactorial para la duración total del amamantamiento muestra el mismo patrón de relación que los modelos unifactoriales, con excepción del grado de escolaridad y la clase de familia (cuadro 6). Los principales factores predictivos de la duración total del amamantamiento fueron la experiencia previa de haber

CUADRO 3. Modelos unifactoriales de riesgo instantáneo proporcional aplicados a la duración del amamantamiento exclusivo en una muestra de madres $(n=556)$ de barrios pobres de Managua, Nicaragua, 1993

\begin{tabular}{|c|c|c|c|}
\hline Variable & $\begin{array}{l}\text { Coeficiente } b \\
\text { (error estándar) }\end{array}$ & $\begin{array}{l}\text { Riesgo relativo } \\
\qquad(\mathrm{IC} 95 \%)^{\mathrm{a}}\end{array}$ & Valor $P$ \\
\hline \multicolumn{4}{|c|}{ Experiencia de amamantamiento previo } \\
\hline Nula (primípara) & \multicolumn{3}{|c|}{ Grupo de referencia } \\
\hline Nula (multípara) & $0,23(0,20)$ & 1,26 & $-\mathrm{b}$ \\
\hline$<6$ meses & $0,12(0,13)$ & 1,13 & \\
\hline$\geq 6$ meses & $-0,20(0,11)$ & 0,82 & $0,04^{c}$ \\
\hline \multicolumn{4}{|l|}{ Subsidio para leche } \\
\hline No & \multicolumn{3}{|c|}{ Grupo de referencia } \\
\hline Sí & $0,17(0,13)$ & 1,18 & 0,09 \\
\hline Riesgo ambiental & $-0,19(0,01)$ & $0,98^{d}(0,96$ a 1,00$)$ & 0,10 \\
\hline
\end{tabular}

a El intervalo de confianza del 95\% (IC95\%) solo se calculó cuando se realizó una prueba bilateral (de dos colas).

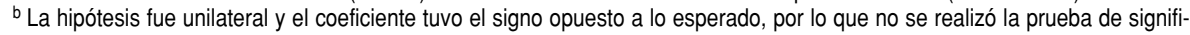
cación.

c Valor $P$ correspondiente a una prueba unilateral.

${ }^{\mathrm{d}}$ Riesgo relativo asociado de cese del amamantamiento por cada unidad de incremento del índice de riesgo ambiental.

amamantado 6 meses o más y la situación laboral, seguidos de las actitudes y el apoyo social (solo el apoyo percibido). Cuando se incluyó la duración del amamantamiento exclusivo en un modelo multifactorial junto con la variable de experiencia previa, disminuyó la intensidad de la asociación con el amamantamiento total, de manera que, en parte, esa relación se explica por la experiencia previa.

\section{DISCUSIÓN Y CONCLUSIONES}

En la presente investigación se utilizó una metodología combinada para estudiar la duración del amamantamiento en una población urbana pobre. El análisis múltifactorial permitió cuantificar relativamente la importancia de las variables relacionadas con la duración del amamantamiento. Los resultados de la discusión de los grupos focales se emplearon para interpretar los resultados de la encuesta en un marco más completo que el del mero análisis cuantitativo.

El patrón de alimentación del lactante en esta población puede describirse como una tasa relativamente elevada de inicio del amamantamiento, la inexistencia casi total de amamantamiento exclusivo y el destete de $30 \%$ de los bebés a las 12 semanas. Aunque este patrón es similar al hallado en otros estudios en Managua, revela lo mucho que falta para que se alcancen las recomendaciones del Ministerio de Salud de Nicaragua, que aconseja 6 meses de amamantamiento exclusivo. La elevada tasa de iniciación hallada es coherente con las discusiones de los grupos, que revelaron tanto una actitud favorable hacia el amamantamiento como los estímulos externos que las madres reciben para seguir esa práctica. Sin embargo, la percepción del amamantamiento exclusivo no parece ser muy favorable, ya que se considera insuficiente para el niño y perjudicial para la salud materna. Patrones similares de alimentación del lactante y tasas bajas de amamantamiento exclusivo se han hallado también en otras poblaciones urbanas (24-27). 
Contrariamente a lo previsto, los resultados del análisis multifactorial indicaron que la duración del amamantamiento exclusivo no es un factor predictivo del amamantamiento total. Esto parece coherente con los resultados obtenidos en los grupos de discusión. Es poco probable que el amamantamiento exclusivo, siendo tan infrecuente, sea un factor predictivo importante del período total de amamantamiento. En los grupos de discusión se observó que, independientemente de la suplementación, algunas madres tenían más éxito que otras en el amamantamiento prolongado, diciendo que su hijo "prefería el pecho al biberón". Las madres que habían destetado más precozmente decían que su hijo "rechazaba el pecho". Esto indica que en una población en la que el amamantamiento exclusivo es raro, el tipo de suplementos, la frecuencia de la alimentación y el vehículo empleado (biberones o taza y cuchara) pueden ser factores más importantes para la determinación del amamantamiento total. Otros autores han indicado que independientemente de que usen o no biberón, algunas mujeres tienen más éxito en amamantar a sus hijos por períodos prolongados (25). En un estudio de una población urbana de México se observó que el amamantamiento exclusivo al mes y a los 3 meses pronosticaba una menor tasa de destete entre el mes y los 3 meses de edad y entre los 3 y los 6 meses, respectivamente (15).

La asociación directa entre las actitudes y la duración del amamantamiento es coherente con la literatura científica sobre lactancia materna (15, 28). Es importante conocer las actitudes respecto al amamantamiento para planear intervenciones, porque puede que el cambio de comportamiento exija la modificación de algunas actitudes existentes, el refuerzo de otras, o ambas cosas. Este estudio ha mostrado que en esta cultura las actitudes hacia el amamantamiento en general son distintas de las mostradas con respecto al amamantamiento exclusivo. Una observación importante derivada de los grupos de discusión es que una actitud favorable hacia el
CUADRO 4. Modelos multifactoriales de riesgo instantáneo proporcional aplicados a la duración del amamantamiento exclusivo en una muestra de madres de barrios pobres de Managua, Nicaragua, 1993

\begin{tabular}{|c|c|c|c|}
\hline Variable & $\begin{array}{l}\text { Coeficiente } b \\
\text { (error estándar) }\end{array}$ & $\begin{array}{l}\text { Riesgo relativo } \\
\quad(\text { IC95\%) }\end{array}$ & Valor $P$ \\
\hline \multicolumn{4}{|c|}{ Experiencia previa en amamantamiento } \\
\hline Nula (primípara) & \multicolumn{3}{|c|}{ Categoría de referencia } \\
\hline Nula (multípara) & $0,25(0,20)$ & 1,29 & $\sim^{b}$ \\
\hline$<6$ meses & $0,10(0,13)$ & 1,10 & $-{ }^{\mathrm{b}}$ \\
\hline$\geq 6$ meses & $-0,18(0,11)$ & 0,83 & 0,05 \\
\hline \multicolumn{4}{|l|}{ Subsidio para leche } \\
\hline No & \multicolumn{3}{|c|}{ Categoría de referencia } \\
\hline Sí & $0,14(0,13)$ & 1,15 & $0,14^{\mathrm{c}}$ \\
\hline Riesgo ambiental & $-0,015(0,01)$ & $0,98^{d}(0,96$ a 1,01$)$ & 0,20 \\
\hline
\end{tabular}

amamantamiento no impide una actitud favorable respecto a la alimentación con biberón. Ambas actitudes se hallaron juntas. En los grupos se reve-

laron diversas actitudes que obstaculizaban el amamantamiento exclusivo, pero no motivaciones favorables específicas. Quizá los futuros estudios cua-

CUADRO 5. Modelos unifactoriales de riesgo instantáneo proporcional aplicados a la duración total del amamantamiento en una muestra de madres de barrios pobres de Managua, Nicaragua, 1993

\begin{tabular}{|c|c|c|c|}
\hline Variable & $\begin{array}{l}\text { Coeficiente } b \\
\text { (error estándar) }\end{array}$ & $\begin{array}{l}\text { Riesgo relativo } \\
\quad(\text { IC95\%) }\end{array}$ & Valor $P$ \\
\hline Duración del amamantamiento exclusivo & $-0,05(0,02)$ & $0,95^{b}$ & $0,03^{c}$ \\
\hline \multicolumn{4}{|l|}{ Nivel de escolaridad } \\
\hline Analfabeta & & Grupo de referencia & \\
\hline Educación elemental & $0,12(0,29)$ & $1,12(0,64$ a 1,97$)$ & 0,69 \\
\hline Educación secundaria como mínimo & $0,53(0,28)$ & 1,71 & 0,06 \\
\hline \multirow{2}{*}{\multicolumn{4}{|c|}{$\begin{array}{l}\text { Experiencia previa en amamantamiento } \\
\text { Nula (primípara) }\end{array}$}} \\
\hline & & & \\
\hline Nula (multípara) & $-0,16(0,31)$ & 0,86 & 0,31 \\
\hline$<6$ meses & $0,25(0,18)$ & 1,29 & $-^{d}$ \\
\hline$\geq 6$ meses & $-1,18(0,21)$ & 0,31 & $<0,001^{c}$ \\
\hline Autoeficacia & $0,03(0,02)$ & 1,03 & \\
\hline Actitudes & $-0,14(0,05)$ & $0,87^{e}$ & $0,01^{c}$ \\
\hline \multicolumn{4}{|l|}{ Situación laboral } \\
\hline Ama de casa & & Grupo de referencia & \\
\hline Con trabajo & $0,43(0,17)$ & 1,54 & $0,01^{\mathrm{c}}$ \\
\hline \multicolumn{4}{|l|}{ Apoyo social } \\
\hline \multicolumn{4}{|l|}{ Tipo de familia } \\
\hline Familia nuclear & & Grupo de referencia & \\
\hline Familia extensa & $-0,41(0,16)$ & 0,67 & 0,01 \\
\hline \multicolumn{4}{|l|}{ Apoyo percibido } \\
\hline No & & Grupo de referencia & \\
\hline Sí & $-0,51(0,16)$ & 0,60 & $0,001^{c}$ \\
\hline
\end{tabular}


CUADRO 6. Modelo multifactorial de riesgo instantáneo proporcional aplicado a la duración total del amamantamiento en una muestra de madres de barrios pobres de Managua, Nicaragua, 1993

\begin{tabular}{|c|c|c|c|}
\hline Variable & $\begin{array}{l}\text { Coeficiente } b \\
\text { (error estándar) }\end{array}$ & $\begin{array}{l}\text { Riesgo relativo } \\
(\text { IC95\%) }\end{array}$ & Valor $P$ \\
\hline Duración del amamantamiento exclusivo & $-0,015(0,024)$ & $0,98^{b}$ & $0,26^{c}$ \\
\hline \multirow{2}{*}{\multicolumn{4}{|c|}{$\begin{array}{l}\text { Experiencia previa en amamantamiento } \\
\text { Nula (primípara) }\end{array}$}} \\
\hline & \multicolumn{3}{|c|}{ Grupo de referencia } \\
\hline Nula (multípara) & $-0,08(0,32)$ & 0,93 & 0,41 \\
\hline$<6$ meses & $0,28(0,18)$ & 1,33 & $-d$ \\
\hline$\geq 6$ meses & $-1,15(0,22)$ & 0,32 & $<0,01$ \\
\hline Actitudes & $-0,11(0,05)$ & $0,90^{e}$ & $0,02^{\mathrm{C}}$ \\
\hline \multicolumn{4}{|l|}{ Situación laboral } \\
\hline Ama de casa & \multicolumn{3}{|c|}{ Grupo de referencia } \\
\hline Empleada & $0,46(0,17)$ & 1,58 & $0,02^{c}$ \\
\hline \multicolumn{4}{|l|}{ Apoyo social } \\
\hline \multicolumn{4}{|l|}{ Tipo de familia } \\
\hline Nuclear & \multicolumn{3}{|c|}{ Grupo de referencia } \\
\hline Extensa & $-0,21(0,16)$ & 0,81 & $0,09^{c}$ \\
\hline \multicolumn{4}{|l|}{ Apoyo percibido } \\
\hline No & \multicolumn{3}{|c|}{ Grupo de referencia } \\
\hline Sí & $-0,35(0,16)$ & 0,71 & $0,02^{\mathrm{c}}$ \\
\hline
\end{tabular}

a El intervalo de confianza del 95\% (IC95\%) solo se calculó cuando se realizó una prueba bilateral (de dos colas).

${ }^{b}$ Riesgo relativo asociado con el cese del amamantamiento por cada unidad de incremento en la duración del amamantamiento exclusivo.

c Valor $P$ correspondiente a una prueba unilateral.

d La hipótesis fue unilateral y el coeficiente tuvo el signo opuesto a lo esperado, por lo que no se realizó la prueba de significación.

${ }^{\text {e }}$ Riesgo relativo asociado con el cese del amamantamiento por cada unidad de incremento de la escala de actitudes.

litativos deban centrarse en las madres que practican exitosamente el amamantamiento exclusivo, para detectar los factores responsables de que esas mujeres sean diferentes.

En la encuesta, el apoyo social se determinó según el tipo de familia y la percepción que tenía la madre del apoyo recibido. En los grupos de discusión se señaló que las madres $\mathrm{y}$ otras mujeres mayores tienen una función importante como asesoras en temas de amamantamiento, lo que posiblemente explique esta relación. No obstante, otra conclusión derivada de los grupos es que las madres necesitan otras clases de apoyo social, dados los obstáculos materiales para el amamantamiento. En la comunidad se pueden superar algunos de esos obstáculos si se da ayuda material e instrumental a las madres por medio de grupos de apoyo. En estudios futuros conviene examinar la estructura de apoyo social con más profundidad, sobre todo en lo referente a ciertos obstáculos materiales al amamantamiento.
El trabajo fuera del hogar fue un factor predictivo importante. En los grupos de discusión las madres trabajadoras indicaron que es necesario dar pronto el biberón para preparar al niño para cuando ellas comiencen otra vez a trabajar fuera del hogar. Las madres consideraron también que el trabajo constituye una dificultad para el amamantamiento. Algunas madres pueden llevar a su bebé al centro de trabajo, pero muchas trabajadoras de Managua tienen que recorrer grandes distancias en la ciudad, con transporte inseguro y otros problemas que las alejan del lactante más de 8 horas al día. En estudios previos ya se observó que las madres nicaragüenses citan el trabajo como razón para dejar de amamantar a sus hijos, introducir la alimentación en biberón o destetarlos por completo $(3,5,29)$.

La literatura científica presenta diversas valoraciones de la relación amamantamiento-trabajo. Según Van Esterik (30), en algunos estudios no se observa que el empleo de la madre afecte a la duración del amamanta- miento, mientras que otros estudios indican lo contrario. Más recientemente, Winikoff y Laukaran (25) y Van Esterik (31) han indicado que el trabajo en sí no es un factor determinante importante de la conducta referente al amamantamiento. Las diferencias observadas en distintos estudios probablemente se deben a la distinta participación de las mujeres en la fuerza laboral y a las condiciones particulares de las trabajadoras en cada país o territorio.

Las participantes en los grupos de discusión indicaron que el personal de los servicios de salud comunitarios favorece el amamantamiento, aunque en la encuesta esta variable (el contacto con los servicios de salud) no fue importante. Los grupos de discusión indicaron al menos que las madres son receptivas a los mensajes de médicos y enfermeras, aunque parece que esos mensajes son muy generales y no siempre ofrecen la respuesta correcta a los problemas.

Con excepción de la experiencia previa, las variables de la encuesta relacionadas con la duración del amamantamiento total no guardaron relación con la duración del amamantamiento exclusivo. Este resultado no es compatible con la hipótesis de que las mismas variables inciden en ambos factores, pero sí con la falta de una cultura de amamantamiento exclusivo demostrada por los grupos de discusión.

En los datos de la encuesta la falta de relación entre la autoeficacia y la duración del amamantamiento fue un resultado inesperado. La discusión en los grupos sugirió una función importante de la falta materna de autoeficacia, ya que las madres creían que su leche no era suficiente para alimentar al niño. Algunas también decían que una madre no puede amamantar a su niño si su alimentación es deficiente. Una posible explicación de la falta de asociación en los resultados de la encuesta es que la medida de autoeficacia se centró en la determinación de la capacidad de dar atención y cuidados maternos que tienen las mujeres en general. La escala no se había diseñado para medir en concreto la autoeficacia para amamantar. Dada la discusión en los grupos, la capacidad de dar aten- 
ción y cuidados que posee una madre no tiene por qué asociarse con su autoeficacia para amamantar. cesita más investigación para aprender a promover el amamantamiento exclusivo y apoyar a las madres en esta práctica. Si como indica este estudio, en esta población el amamantamiento exclusivo no se considera posible ni bueno para la madre, es probable que las madres no escuchen los mensajes de los programas de promoción actualmente en marcha. Los trabajadores de salud quizá estén hablando un idioma "distinto" al de la población destinataria. Se necesitan programas específicos para crear una imagen favorable del amamantamiento exclusivo.
Los resultados muestran que se ne-

Las madres sin experiencia previa en amamantamiento son un blanco importante de las actividades de promoción y apoyo. Este estudio ha confirmado también la necesidad de políticas destinadas a apoyar a la mujer trabajadora que amamanta a sus hijos.

Agradecimiento. Expresamos nuestro agradecimiento a Leonel Argüello por el apoyo recibido en Managua durante el período de recogida de datos; a Patrice Engle por su amabilidad al compartir la escala de cuantificación de la autoeficacia; a Eleanor Parker por su ayuda en el procesamiento de textos y a Jeanine Castro y Patricia Carrillo por su colaboración en los grupos de

\section{REFERENCIAS}

1. Saadeh R, ed. Breast-feeding: the technical basis and recommendations for action. Geneva: World Health Organization; 1993.

2. Cameron M, Hofvander Y. Manual on feeding infants and young children. Oxford: Oxford University Press; 1983.

3. De Macías O'Leary G. Case study: a women's movement in Nicaragua, an advocate in breastfeeding. Assignment Children 1981:55/66.

4. Nicaragua, Ministerio de Salud, Dirección Maternoinfantil. Efecto del acercamiento temprano madre-infante sobre la incidencia y duración de la lactancia materna. Managua: MINSA; 1983. (Documento inédito).

5. Osorio E, Solórzano L. Incidencia y duración de la lactancia materna durante un período de tres meses en el área de salud Carlos Rugama de Managua [tesis]. Managua: Universidad Centroamericana; 1988.

6. Centro de Investigación y Estudios sobre la Salud. Enfoque de riesgo y estado nutricional de los niños menores de cinco años en la región III. Managua: CIES; 1988. (Documento inédito).

7. Stupp P, Monteith R, Cuadra R, Whittle L. Encuesta sobre salud familiar, Nicaragua 92-93. Managua: Profamilia; 1993.

8. Allen LH, Pelto GH. Research on determinants of breastfeeding duration: suggestions for biocultural studies. Med Anthropol 1985; 9:97-105.

9. Greene J, Caracelli V, Graham W. Toward a conceptual framework for mixed-methods evaluation designs. Educ Eval Pol Anal 1989; 11:255-274.

10. Perry CL, Baranowski T, Parcel G. How individuals, environments, and health behaviour interact: social learning theory. En: Glanz K, Lewis FM, Rimer BK, eds. Health behavior and health education. San Francisco: Jossey-Bass; 1990:161-186.
11. Parcel GS, Baranowski T. Social learning theory and health education. Health Educ 1981; 12:14-18.

12. Engle P, LaMontagne, JF, Zeitlin M. Caring capacity within the household and nutritional status: an observational study of caring behaviors. Final report. Managua; 1992. (Documento inédito).

13. Carmines EG, Zeller RA. Reliability and validity assesment. Newbury Park, California: Sage; 1991.

14. Simss LS. Toward an understanding of attitude assesment in nutrition research. $J \mathrm{Am}$ Diet Assoc 1981;78:460-466.

15. Stewart KA. Psycho-social and behavioral predictors of breastfeeding duration in an urban Mexican population [tesis de maestría]. Ithaca: Cornell University; 1989.

16. Dawson-Saunders B, Trapp RG. Basic and clinical biostatistics. Norwalk, CO: Appleton and Lange; 1990.

17. Friedman L, Furberg C, DeMets D. Fundamentals of clinical trials. Saint Louis, Missouri: Mosby-Year Book; 1985.

18. Christensen E. Multivariate survival analysis using Cox's regression model. Hepatology 1987;7:1346-1358.

19. Dean AG, Dean JA, Burton AH, Dicker RC. Epi Info, version 5-A word processing, database and statistics program for epidemiology on microcomputers. Stone Mountain, Georgia: USD; 1990.

20. SPSS/PC +4.0 Base manual. Chicago, Illinois: SPSS; 1990.

21. SYSTAT for Windows: DATA, Version 5 Edition. Evanston, Illinois: Systat; 1992.

22. Steinbers D, Colla P. SURVIVAL: a supplementary module for SYSTAT. Evanston, Illinois: Systat; 1988.

23. Guba EG, Lincoln YS. Fourth generation evaluation. Newbury Park, California: Sage; 1989. discusión. La primera autora tiene una deuda de gratitud con Jennifer Greene, que formó parte de su Comité Especial. El estudio se financió con fondos del Van Veen International Travel Grant, por mediación de la División de Ciencias de la Nutrición de la Universidad Cornell, la Representación de la OPS en Managua y el Instituto de Nutrición de Centro América y Panamá (INCAP). El trabajo de Janet Irene Picado fue remunerado en parte por una beca de capacitación (T32DK07158) de los Institutos Nacionales de Salud de los Estados Unidos, concedida a la División de Ciencias de la Nutrición de la Universidad Cornell.
24. O'Gara C, Kendall C. Fluids and powders: options for infant feeding. Med Anthropol 1985; 9:107-122.

25. Winikoff B, Laukaran VH. Breast feeding and bottle feeding controversies in the developing world: evidence from a study in four countries. Soc Sci Med 1989;29:859-868.

26. Pérez Escamilla R. Breast feeding patterns in nine Latin American and Caribbean countries. Bull Pan Am Health Organ 1993;27:32-42.

27. Dimond HJ, Ashworth A. Infant feeding practices in Kenya, Mexico and Malaysia: the rarity of exclusive breastfeeding. Hum Nutr Appl Nutr 1987;41A:51-64.

28. Jones DA. Attitudes of breastfeeding mothers: a survey of 649 mothers. Soc Sci Med 1986; 23:1151-1156.

29. Cattaneo A. Vol. VI. Prevalencia y duración de la lactancia materna en la ciudad de León. Nicaragua: Sistema de Vigilancia Epidemiológica Nutricional; 1990.

30. Van Esterik P. Women, work and breastfeeding. New York: Cornell University; 1992. (Cornell International Nutrition Monograph Series Number 23).

31. Van Esterik P. Beyond the breast bottle controversy. New Brunswick, New Jersey: Rutgers University Press; 1989.

Manuscrito recibido el 28 de julio de 1995 y aceptado para publicación en versión revisada el 23 de enero de 1997. 
ABSTRACT This study examined the relationship of several maternal variables to the duration of exclusive breast-feeding and the total duration of breast-feeding, along with attitudes, perceptions, and beliefs about breast-feeding among women living in poor neighborhoods of Managua, Nicaragua. The field work was carried out in December 1992 and January 1993 using qualitative and quantitative methods. A structured questionnaire was administered in interviews with 556 mothers of children under 12 months of age, and meetings of four directed discussion groups were held, in which a total of 20 women participated. At one week of age, almost all the children of the mothers who were surveyed had been breast-fed, but only $45 \%$ had been exclusively breast-fed. At 12 weeks old, $30 \%$ were already completely weaned. The discussion groups revealed the coexistence of positive opinions about both breast-feeding and bottle-feeding. However, exclusive breast-feeding was considered harmful for the mother, and breast milk was not thought to be sufficient nourishment for the child. Previous experience was strongly related to the duration of exclusive breast-feeding and to total breastfeeding duration. Attitudes, social support, and work situation were important factors influencing the total length of time women breast-fed a child. In general, the results obtained through the interviews and in the discussion groups were in agreement and showed that the elements needed to promote exclusive breast-feeding were social support, a favorable community environment, and policies that dealt with problems faced by working mothers. The complementary research methods were useful for obtaining information about the relative importance of different factors that determine the duration of breast-feeding and for understanding that practice in greater depth from the mother's point of view.

\title{
41. ${ }^{a}$ Reunión Anual del Council of Biology Editors
}

Fechas: 1 a 5 de mayo de 1998

Lugar: Salt Lake City, Utah, Estados Unidos de América

Durante su $41 .^{a}$ reunión anual, al igual que durante sus reuniones anteriores, el Council of Biology Editors ofrece a sus afiliados y a personas sin afiliación pero dedicadas a la edición científica un programa de enfoque didáctico sobre diferentes aspectos de la labor editorial. Habrá discusiones en grupo y talleres prácticos para editores y administradores de revistas científicas y para redactores de trabajos de investigación. "La comunicación científica en el siglo XXI" y "La transmisión al público de los resultados de la investigación científica" serán los temas tratados en dos sesiones plenarias. También habrá oportunidad de participar en actividades turísticas y de recreo.

\author{
Información: \\ Andrea Blood o Denise Baran \\ 60 Revere Drive, Ste. 500 \\ Northbrook, IL 60062 EUA \\ Tel: (847) 480-9080 \\ Fax: (847) 480-9282 \\ E-mail: cbehdqts@aol.com \\ Sitio en la WWW: http://www.cbe.org/cbe
}

